\title{
Coulisses
}

Revue de théâtre

\section{Les didascalies dans le théâtre d'Edmond Rostand : entre verbe et action poétique}

\section{Géraldine Vogel}

\section{(2) OpenEdition}

1 Journals

Édition électronique

URL : https://journals.openedition.org/coulisses/979

DOI : 10.4000/coulisses. 979

ISSN : 2546-9460

\section{Éditeur}

Presses universitaires de Franche-Comté

\section{Édition imprimée}

Date de publication : 31 décembre 2009

Pagination : 87-100

ISBN : 978-2-84867-270-0

ISSN : $1150-594 X$

\section{Référence électronique}

Géraldine Vogel, «Les didascalies dans le théâtre d'Edmond Rostand : entre verbe et action poétique », Coulisses [En ligne], 39 | Automne 2009, mis en ligne le 30 novembre 2016, consulté le 29 décembre 2022. URL : http://journals.openedition.org/coulisses/979; DOI : https://doi.org/10.4000/coulisses 979

Ce document a été généré automatiquement le 29 décembre 2022.

Tous droits réservés 


\title{
Les didascalies dans le théâtre d'Edmond Rostand : entre verbe et action poétique
}

\author{
Géraldine Vogel
}

\section{Introduction}

La seconde moitié du XIX siècle voit naître le naturalisme au théâtre et, avec lui, les balbutiements de la mise en scène, chère à Antoine. Le personnage dramatique, plus individualisé, tente de s'affranchir de son milieu. Par conséquent, les didascalies ${ }^{1}$, miroir de l'environnement dans lequel l'homme évolue, gagnent en importance dans les textes théâtraux de la Belle-Époque. Tout comme certains de ses contemporains, Edmond Rostand a abondamment recours aux indications scéniques dans ses huit pièces de théâtre. Il paraît ainsi essentiel de se demander comment il les manipule et quel but elles servent. Afin de comprendre le fonctionnement des didascalies dans le théâtre rostandien, il s'avère nécessaire de considérer, dans un premier temps, la fréquence de leur apparition puis, leur relation avec les répliques des personnages centraux. Dans un troisième temps, l'analyse de la mise en place du décor par les indications scéniques en début d'acte permettra de mesurer leur place dans des pièces qui, pour la plupart, sont centrées sur la construction d'une " attitude » poétique chez des protagonistes qui échappent à leur milieu.

\section{La « glose » didascalique : une mise en abîme de la création poétique}

2 Tout d'abord, l'auteur de Cyrano de Bergerac utilise pleinement la didascalie fonctionnelle pour indiquer les lieux et les époques qui jalonnent les pièces (sauf, bien entendu, dans le cas de Chantecler, pièce atemporelle) - le décor et la disposition de la scène, la musique et les jeux de lumière, les apartés et les "voix hors-champs ", les 
mouvements et les sentiments des personnages. Les indications scéniques vont même parfois jusqu'à préciser d'apparentes évidences comme dans Chantecler, par exemple, où le spectateur et le lecteur attendent du Chat-Huant qu'il poursuive naturellement sa réplique. Or Rostand précise : « Le Chat-Huant reprenant la parole » et « Le Chat-Huant, terminant sa phrase $»^{2}$.

3 Certaines de ces didascalies fonctionnelles vont même jusqu'à se répéter. Dans la même pièce, une note sur la place d'un personnage qui figure dans les didascalies écrites directement entre le titre du premier acte et l'annonce de la première scène apparaît également au moment où sont énumérés les personnages qui vont paraître. «Un merle dans sa cage » devient « le Merle dans sa cage qui est accrochée parmi les glycines » un peu plus loin $^{3}$. La minutie extrême de Rostand, qui ne cesse de développer et de commenter ses dialogues, témoigne sans doute d'une crainte d'auteur: que les destinataires interprètent mal son œuvre. A ce sujet, Patrice Pavis remarque que lorsque les indications scéniques fonctionnelles et initiales sont abondantes dans un texte de théâtre, "[c]e texte veut noter sa future mise en scène $»^{4}$. Plus le poète dramatique commente son dialogue théâtral, plus l'éventail d'interprétations du metteur en scène et du lecteur s'en trouve restreint. Rostand est-il semblable à son Cyrano dont le «sang se coagule/En pensant qu'on peut changer une virgule [à ses œuvres littéraires] $»^{5}$ ? C'est ce qu'estime Pierre Mortier dans l'entrevue avec Rostand dont il rend compte pour Je sais tout. Il explique, en reprenant les paroles du dramaturge :

C'est lui-même [Rostand] qui dessine les costumes et les maquettes de ses décors. Il veut que rien ne soit livré au hasard, que tout soit prévu et établi à l'avance, et il ne peut s'empêcher de nous dire combien il méprise les faiseurs de pièces qui comptent sur leurs interprètes pour avoir du succès. «Le devoir d'un comédien est de traduire fidèlement, servilement, la pensée de l'auteur, de se départir presque de toute personnalité, de s'effacer complètement derrière le texte, et le plus grand artiste est celui qui observe le mieux ces principes. $»^{6}$

Après de telles prises de position, il n'est plus étonnant de constater que Rostand se montra très actif dans la mise en scène de ses pièces et n'eut recours que très rarement à des metteurs en scène extérieurs. Il dirigeait sévèrement, de près ou de loin, les répétitions de ses pièces. En effet, lorsque Rostand ne pouvait rejoindre Paris au moment des répétitions de Chantecler, en 1909 et en 1910, il entretint une correspondance détaillée avec le directeur du Théâtre de la Porte Saint-Martin, Jean Coquelin, dans le but d'assurer une mise en scène des plus méticuleuses pour cette pièce déjà, en soi, difficile à monter. Comme celle de Chantecler, la toute première mise en scène de Cyrano de Bergerac témoigne du même souci de régularité. Dans une lettre adressée à Constant Coquelin, l'interprète du personnage principal, l'auteur dramatique ajoute soigneusement des directives sur le jeu des acteurs, comme si les didascalies du texte d'origine ne devaient pas suffirE ${ }^{7}$. Ainsi, ces deux lettres peuvent être considérées comme des «indications scéniques à retardement» destinées à combler certaines lacunes du texte de départ.

$5 \quad$ Les manuscrits d'origine de Cyrano de Bergerac et de Chantecler étant introuvables, il est difficile d'étudier les modifications didascaliques subies par la mise en travail de la pièce sur la scène. Toutefois, une traduction du Faust de Goethe par Rostand comporte diverses précisions n'existant pas dans le texte allemand: on y trouve des indications scéniques supplémentaires, surtout en début d'actes, ajoutées avant la mise en répétition. Même si les manuscrits d'origine - celui de Faust en particulier - attestent 
de l'ajout de didascalies « de travail », il ne fait aucun doute que d'autres détails furent ajoutés sur épreuve, pour le bénéfice du lecteur, signe que Rostand, à la fin du processus créatif, voyait le théâtre avec l'œil du spectateur's. Or, matériellement, le public fait en effet d'abord l'expérience de la mise en scène, ce qui permet à l'auteur de voir et de corriger les défauts du manuscrit. Dans le souci d'une mise en scène méticuleuse et fidèle aux idées de sa pièce, pour l'acte intitulé «La Rôtisserie des poètes » dans Cyrano de Bergerac, Rostand fit commander du jambon, idée qu'il rajouta sans doute au manuscrit de la pièce, une fois son succès assuré9. «[...] ce [...] jour où, l'avant-veille de la générale, il fallut, pour que la rôtisserie prît tout de même l'air d'être un peu vivante, aller chercher de vrais saucissons et un vrai jambon et un vrai pâté pour réveiller ceux qui dormaient sur du carton! » rappelle Rosemonde Gérard ${ }^{10}$. En plus d'accorder une grande importance à sa propre vision du poème dramatique, Edmond Rostand est effectivement tenaillé par le souci d'exactitude dans la représentation du réel car, loin d'être uniquement poétique, son théâtre est également, de par la mise en scène, naturaliste.

Outre l'obsession de faire vrai et donc de rendre son théâtre plus objectif, l'auteur, qui éprouve le besoin de préciser les moindres actes et gestes de ses personnages, les moindres modifications spatio-temporelles, " vit » sa pièce, la voit se dérouler sous ses yeux. A ce propos, le journaliste Pierre Mortier affirme : "Lorsqu'il [Rostand] fait une pièce, il la joue à mesure. Dans son esprit, les héros agissent et se meuvent; il a dans les yeux tous leurs gestes, dans l'oreille toutes leurs intonations. Il peut mettre soixante personnages en scène (c'est le cas de Chantecler), il vit successivement de la vie de chacun [...]. $»^{11}$ Cette remarque met en relief la volonté de Rostand de maîtriser le geste et la parole, et pour transmettre ce qu'il voit au lecteur et au public, il emprunte une voix narrative semblable à celle du récit, une voix qui, finalement, annule la spécificité scénique du théâtre. L'ajout d'un titre à chaque acte de La Samaritaine, de Cyrano de Bergerac, de L'Aiglon et de Chantecler semble appuyer cette constatation. Ces actes sont à voir comme des chapitres de roman ou même de courts romans, ou de brèves nouvelles autonomes. Or, si les didascalies rostandiennes rappellent le roman de par leur aspect descriptif, ceci veut-il pour autant dire qu'elles obscurcissent le dialogue théâtral, qu'elles l'enferment dans des gloses interminables?

7 Certes, il est possible de les considérer comme des « gloses » vis-à-vis de l'intervention des personnages, et ceci dans le sens où elles se rajoutent au dialogue pour le compléter et, surtout, l'expliciter. Les indications scéniques permettent ainsi de dissiper toute ambiguïté sur les intentions des personnages comme, dans Chantecler, la fausseté du Merle qui "feint de vouloir empêcher Chantecler de passer" lorsque ce dernier souhaite rejoindre le combat contre son adversaire, le Pile Blanc, engagé par les Nocturnes pour l'assassiner ${ }^{12}$. En précisant les desseins des protagonistes, desseins dont les héros ne sont pas toujours conscients, la didascalie retrouve la fonction du chœur et affirme la présence moralisatrice de l'auteur dans sa pièce. Dans son Discours de Réception à l'Académie Française, Rostand est d'ailleurs jusqu'à un certain point conscient de son " interventionnisme », comme il le reconnaît ci-dessous :

Je n'ai jamais été plus tenté de ne pas parler en prose. Au moment d'entreprendre ce discours, j'aurais volontiers recouru, pour me donner de la hardiesse, à une fiction d'auteur dramatique. Il m'eût été commode d'imaginer que j'écrivais une pièce dans laquelle il arrivait à un tout indigne poète ce qui vient, paraît-il, de m'arriver; et vous conviendrez, messieurs, qu'une pièce où il y a de ces invraisemblances ne saurait être qu'en vers. [...] C'était du théâtre héroïque ; j'y 
aurais peut-être réussi ; j'aurais eu pour mon héros de plus abondantes bravoures que pour moi-même ; et j'aurais fait mon discours en croyant faire le sien. Mais j'ai pris garde que je ne devais pas chercher à me faciliter l'épreuve ; je m'abstiendrai donc du langage qui m'est le moins étranger, encore qu'il soit peu raisonnable de doubler les émotions, et de vouloir débuter ensemble sous la Coupole et dans la prose. $^{13}$

De son propre aveu, les personnages qu'il met en scène racontent donc jusqu'à un certain point l'histoire romancée de ses propres passions et de sa propre vie. La pièce correspond ainsi plus à une biographie de poète qu'à une autobiographie de leur auteur. C'est à dessein que le poète dramatique, dont la voix est présente dans chacune des paroles de ses figures poétiques, a recours aux indications scéniques pour compléter ce qui doit figurer sur la scène car il n'est de meilleur conteur que celui qui a vécu l'expérience dont il témoigne. Cette constatation est tellement évidente pour Rostand qu'il semble substituer sa voix propre à celle de ses personnages, dans les didascalies du moins. Mais une analyse plus précise des pièces rostandiennes permet de nuancer cette affirmation, comme dans le premier acte de Cyrano de Bergerac, par exemple, où le personnage central vole la voix du dramaturge, dans le sens où il fournit lui-même les didascalies qui mettent en scène son existence. Comment ne pas voir en effet que la « Ballade du duel qu'en l'hôtel bourguignon/Monsieur de Bergerac eut avec un bélître", n'est qu'une longue indication scénique qui commence avec: "Je jette avec grâce mon feutre, / Je fais lentement l'abandon / Du grand manteau qui me calfeutre, / Et je tire mon espadon [...] $\aleph^{14}$ ? Ainsi, la voix de Cyrano de Bergerac recouvre celle de Rostand.

En effet le poète-bretteur invente et crée sa vie comme il le ferait pour un roman, une pièce de théâtre ou un poème. L'intrigue de Cyrano de Bergerac, c'est-à-dire le souhait qu'ont Christian et Cyrano de gagner l'amour de Roxane, se résume en cette phrase significative : "Toi, du charme physique et vainqueur, prête-m'en : / Et faisons à nous deux un héros de roman. ${ }^{15}{ }^{15}$ 'est ce héros de roman que le protagoniste s'efforce de mettre en scène tout au long de la pièce dans laquelle il se crée un rôle. De cette manière, Rostand montre au lecteur comme au spectateur la manière dont travaille un poète, un romancier, un dramaturge, et, en un mot, un écrivain qui joint la parole aux gestes et le dialogue à la description. A l'instar de Cyrano de Bergerac, Chantecler, le grand coq dont le métier est de chanter l'aurore, décrit sa " démarche " poétique et les gestes qui l'accompagnent à l'aide d'une description qui aurait également pu figurer dans les indications scéniques. "Attends! ... Constate encor qu'impatient et fier / Et grattant le gazon de mes griffes, j'ai l'air / De chercher dans le sol, tout le temps quelque chose... [...] Je ne chante jamais que lorsque mes huit griffes / Ont trouvé, sarclant l'herbe et chassant le caillou, / La place où je parviens jusqu'au tuf noir et doux!", explique le $\operatorname{coq}^{16}$. Ainsi, les paroles du personnage central font écho aux didascalies de Rostand, didascalies qui mettent également en relief une gestuelle propice à la création poétique. Tout comme cet auteur de la Belle-Epoque, le personnage central «vit » sa création, qu'il explique d'une manière quasi-naturaliste aux spectateurs. Les didascalies rostandiennes et les paroles des figures poétiques se répondent ainsi pour mettre en relief la création de deux poèmes, fabriqués simultanément par deux poètes, l'un extérieur et l'autre intérieur à l'intrigue. 


\section{La concomitance des gestes et de la parole poétique : la naissance d'une « attitude »}

10 La " glose » didascalique, destinée avant tout dans le théâtre de Rostand à préciser les contours d'une personnalité qui construit un poème, apporte aussi des précisions sur les gestes et les déplacements des personnages, afin de "joi[ndre] le geste à la parole $»^{17}$. La scène du duel en vers au premier acte de Cyrano de Bergerac et d'autres pièces rostandiennes où le dialogue est entrecoupé de didascalies évoquant les mouvements du personnage central en sont des exemples. Ici, au début du premier acte, alors qu'un Fâcheux invective Cyrano et que ce dernier lui répond en alexandrins, les indications scéniques notent: "La main à son épée ", "Montrant son épée ", "marchant sur lui ", "le soufflette» et "Il le retourne par les épaules " ${ }^{18}$. L'intimidation est davantage gestuelle que verbale, jusqu'à ce que le Fâcheux finisse par se sauver. La seconde provocation, de la part d'un Vicomte cette fois, donne lieu à la tirade du nez. Comme s'il avait une épée à la main, le poète-bretteur accule son adversaire, qui se retrouve "suffoqué " à la fin de la récitation. C'est ainsi que les indications scéniques et la tirade se rejoignent pour combattre l'antagoniste et pour insuffler une dynamique à la pièce. Le geste et la parole poétique sont, donc, équivalents et interchangeables, puisque tous deux ont une égale force de persuasion. Les indications scéniques ont ainsi pour fonction immédiate de mettre en scène le poème en voie de création, voire de s'y substituer.

11 De cette manière, un poème écrit par Rostand et intitulé Le Bois sacré fut créé en 1910 au théâtre Sarah Bernhardt sous la forme d'une pantomime, où les gestes accompagnaient et, surtout, émanaient des vers récités par l'acteur Léon Brémont. Le rôle principal y était tenu par Sarah Bernhardt, actrice que le poète dramatique nommait « Reine de l'attitude et Princesse des gestes ${ }^{19}$. Le vers d'un poème dédié à Constant Coquelin affirmait : " Toi, tu poétisais. Ton geste avait du style », étayant également l'hypothèse selon laquelle la plupart des gestes, dans les pièces rostandiennes, deviennent poésie, s'ils sont effectués par un acteur qui "poétise » et qui s'approprie de manière satisfaisante le texte de théâtre ${ }^{20}$.

12 Si le mouvement des acteurs, précisé par les didascalies, peut générer la poésie, l'inverse est également possible. Dans une lettre adressée à Constant Coquelin, Rostand précise : «Votre scène avec Volny, fin du 2, était déjà dans son bon mouvement, l'autre jour. Si vous y arrivez, (j'en doute car il faut beaucoup redire et fixer le début) ne cherchez qu'à vous la bien mettre (à la lui bien mettre, surtout) dans la bouche. Le mouvement en viendra tout seul, quand il saura imperturbablement. $»^{21}$ Ainsi, la dynamique découle de la connaissance du texte. Voilà pourquoi Rostand ne juge généralement pas nécessaire de fournir des indications scéniques pour les tirades et les récitations poétiques de ses personnages. Les paroles des personnages peuvent se suffire à elles-mêmes pour créer le dynamisme d'un mouvement. Par exemple, l'aurore naît, en apparence mais aussi en réalité, grâce aux paroles poétiques de Chantecler qui, tout au long de ses odes pour faire lever le jour, "ordonne" aux techniciens la modification de la lumière qui doit être apportée à la scène. «Flambe ! ... Illumine ! ... Embrase! » sont les premiers mots du coq dans la pièce de Rostand ${ }^{22}$. Ces précisions maintiennent l'illusion dramatique et cela en plein déploiement technique. Alors que le mouvement peut se transformer en poème, la poésie peut elle-même générer le mouvement et contribuer à créer ainsi une « attitude » face à l'existence. 
13 En effet, l'écriture poétique mise en scène dans Cyrano de Bergerac et Chantecler, tout comme le rêve poétique figurant dans La Princesse lointaine attirent l'attention sur l'opposition contre laquelle les protagonistes principaux devront alors se battre. D'ailleurs, la poésie de Cyrano a pour seule protectrice l'épée. Ces deux éléments, arme et poème, semblent indissociables dans la pièce en question puisque, la parole même du personnage est une attaque ou une parade. Lorsque Cyrano "poétise », il raconte ses gestes, gestes qui auraient, sinon, nécessité des didascalies précises. Or, lorsque ce n'est pas Cyrano qui les conte, ce sont d'autres personnages, dont Ragueneau, poète également, qui prend le relais : le duel versifié, le combat «contre cent » à la Porte de Nesle, la récupération de l'écharpe appartenant à De Guiche en plein champ de bataille. L'action que l'on devrait exclusivement retrouver dans les indications scéniques de début d'acte, ou à la suite des répliques, est régulièrement traduite en poème, comme c'est encore le cas pour la recette des tartelettes amandines, récitée en vers par Ragueneau qui, au lieu de cuisiner devant le spectateur, se contente de dire son poème.

Si poésie et gestes sont inséparables et compatibles en tous points, ils débordent tous deux dans le dialogue en alexandrins. Dans La Princesse lointaine, par exemple, l'affrontement ou la confrontation entre Bertrand d'Allamanon, porte-parole du poète Joffroy Rudel, et la princesse, est relaté par Sorismonde, la suivante, puis par la princesse elle-même. Cette série de gestes qui résument en eux-mêmes un comportement poétique, une " attitude », sont couronnés par un geste final qui sera la récitation du poème de Joffroy Rudel à la princesse. Dans cette logique, les tirades et les poèmes des personnages centraux ne sont que purs gestes. «Chanter, c'est ma façon de me battre et de croire ", explique Chantecler mais n'est-ce pas aussi la façon dont les mariniers de Joffroy Rudel et le poète médiéval existent ${ }^{23}$ ?

La parfaite harmonie entre le geste didascalique, parfois contenu dans la parole, et la parole poétique permettent de montrer la figure du poète en pleine action et de la rendre pleinement crédible aux yeux du spectateur. Dans Cyrano de Bergerac, « l'art de la pointe " s'exerce au combat comme dans la création littéraire. Joffroy Rudel de La Princesse lointaine vit son poème, le crée au fur et à mesure qu'il se rapproche de la princesse de ses rêves. Dans l'absolu, le théâtre de Rostand peut ainsi être vu comme une seule grande didascalie : le dire et le faire s'y mélangent de toutes les façons. Dans La Samaritaine, Photine décrit le Christ de la manière suivante : « Nul n'a son éloquence immense et familière, / Et son geste est celui d'ouvrir une volière!", mettant à nouveau en parallèle le geste et l'éloquence poétique ${ }^{24}$.

Dans Chantecler, pièce la plus poétique de Rostand, la commutativité du geste et de la parole poétique se révèle à chaque ligne. Lors de la création de la pièce, dont la mise en scène avait été minutieusement étudiée par Rostand, l'importance des costumes d'oiseaux empêchait le mouvement des acteurs. Cette première mise en scène respectait scrupuleusement les indications scéniques écrites en vers au début des actes. Il va sans dire que la poésie, dans cette pièce de théâtre, devient progressivement un geste à part entière puisque les personnages ne peuvent s'exprimer que par la voix. En ce qui les concerne, les didascalies fonctionnelles se résument à la description de sautillements et de bondissements effectués par les acteurs encombrés de leur costume d'oiseau.

17 Les indications scéniques qui précisaient l'action et les gestes dans Cyrano de Bergerac se sont progressivement au fil des œuvres chargées de remarques sur la tonalité, la parole et la voix des personnages jusqu'à Chantecler. Quelques exemples de didascalies 
consacrées à la voix de Chantecler : «avec une sorte de râle de tendresse », « d'une voix cordiale, à la Basse-Cour ", "reprenant son ton de commandement ", "dans un long roucoulement tendre ", « parlant vers l'horizon », « d'une voix éclatante », « d'une voix de commandement ", "d'une voix solennelle, tout en parant et ripostant ", "lançant son cri le plus éclatant " puis « de sa voix de commandement » et bien d'autres encore montrent la variété de tonalité avec laquelle le coq s'exprime et mettent en relief la puissance de la parole poétique du coq, c'est-à-dire son « attitude » autoritaire ${ }^{25}$.

Tout comme les précisions sur le geste et la parole poétique, le poème et les didascalies sur le décor ne cessent de se répondre comme l'atteste la métaphore filée du «lierre obscur [qui] circonvient un tronc » retrouvée dans la tirade des «non merci » comme dans les didascalies de la scène du balcon dans Cyrano de Bergerac ${ }^{26}$. Elle montre jusqu'à quel point les indications scéniques fonctionnelles faisant état de la vie sentimentale de Cyrano (Christian étant un parasite profitant des talents poétiques de son rival) et les insuccès littéraires, dont témoignent ses paroles poétiques, vont de pair dans la pièce. En passant dans la didascalie fonctionnelle, les gestes accentuent la véracité de la poésie, seule « réalité » acceptable pour les figures poétiques des pièces rostandiennes. Ces gestes-là forment ce que Rostand appelle «le panache", qu'il définit comme: « l'esprit de la bravoure[,] le courage dominant à ce point la situation qu'il en trouve le mot. » Le panache est un geste de bravoure fait verbe, une " attitude » face à l'âpreté de la vie destinée à durer, comme les écrits. Cette "attitude ", le Chat-Huant du deuxième acte de Chantecler ne la possède pas puisqu'il doit sans cesse réaffirmer l'autorité de sa parole. En rassemblant gestes, dont témoignent les didascalies fonctionnelles, et parole poétique, le personnage principal ressort grandi de son affrontement avec l'existence.

Grâce au panache, qui allie mots et gestes didascaliques, les personnages évoluent dans une atmosphère poétique que Rostand crée et qu'ils s'évertuent, eux-mêmes, en tant que poètes, à reproduire. Les indications scéniques de début d'actes dans Chantecler sont écrites en vers et la description qu'elles établissent du décor fait écho aux diverses odes récitées par le coq. La basse-cour décrite par Rostand dans les didascalies à chaque début d'acte est reprise et développée par Chantecler dans son Ode au Soleil et dans son Ode à la Lumière. C'est à dessein que Rostand précise dans la didascalie du début du premier acte : «Les détails vont se préciser au cours de l'acte. $»^{27}$ Ainsi, le personnage poétique évolue en milieu familier, dans une atmosphère qu'il adapte à ses besoins. Il semblerait que la création d'une atmosphère poétique par le dramaturge et par le protagoniste central permette une distanciation systématique de la notion de « condition » telle que développée par Diderot. Au lieu d'être tributaires de leur milieu, la plupart des personnages poétiques se l'approprient et le recréent à leur guise. C'est pour cette raison qu'il importe maintenant d'analyser les didascalies fonctionnelles dans les pièces rostandiennes en rapport avec le décor où évoluent ces « attitudes ».

\section{L'individu poétique contre son milieu social : une histoire de perspective}

Les didascalies fonctionnelles dans les pièces rostandiennes donnent d'abondantes précisions spatio-temporelles au début de chaque acte. C'est dans ce sens qu'elles assurent l'expansion du texte vers le monde extérieur. Premièrement, et cela contrairement aux pièces précédemment citées, l'importance du lieu, dans L'Aiglon, montre l'enfermement de l'individu dans l'Histoire, empêchant l'affirmation de son 
panache. L'Histoire autrichienne n'en finit pas d'écraser les élans du roi de Rome. Dans le premier acte, les didascalies précisent que l'action se déroulera dans une villa banale appartenant à Marie-Louise, villa qui, malgré son insignifiance dans l'action, paraît déjà caractérisée historiquement. Rostand précise : «au second plan, dans une niche, un énorme poêle de faïence, lourdement historié » comme pour signaler, malgré la position secondaire de cet élément de décor, l'imposante influence de l'Histoire dans le drame individuel auquel assistera le spectateur ${ }^{28}$. Ces allusions à une Histoire imposante jalonnent presque tous les actes de L'Aiglon et sont toujours à mettre en relation avec le destin du jeune homme.

21 Ainsi, le château de Schoenbrünn, où se déroulent la plupart des événements du drame, depuis le deuxième jusqu'au sixième acte, est un lieu également chargé d'un lourd passé historique. Les didascalies du deuxième acte précisent ce poids puisqu'il se déroule dans le Salon des Laques, décoré "d'un lourd et somptueux rococo allemand. $»^{29}$ Deux portraits d'ancêtres autrichiens dominent symboliquement la couronne impériale de Napoléon ${ }^{\mathrm{er}}$. Ce salon situé dans les appartements du Duc, montre d'emblée combien le personnage principal sera conditionné par la domination autrichienne. L'Histoire hante ces indications scéniques très précises où le personnage principal a soigneusement rangé, sur les meubles du Salon des Laques, des livres et des objets de couleurs vives tranchant avec le noir environnant rappelant les exploits de son père. Ces objets de guerre, "un bonnet de grenadier français, des épaulettes rouges, un sabre, une gibeline etc. " s'opposent aux traditionnels objets de chasse autrichiens: «des armes de luxe, des cravaches, des fouets de chasse etc.». Tout le passé historique d'un père glorieux et toute la tradition historique de l'Autriche s'opposent mais s'unissent pour écraser de leur poids la personnalité du jeune duc, personnalité qui se replie dans ses lectures, renonçant à vivre sa vie. Ces trois forces, le passé historique français, autrichien et livresque, empêcheront le duc d'affronter de « dire » et de « faire » l'Histoire à venir.

22 En effet, rien de ce milieu passéiste dans lequel vit le jeune homme ne favorise l'élan spontané, indépendant, d'un poète un peu trop faible. Le jeune duc habite une prison dans laquelle rien ne change jamais, puisque tout lui est interdit. La leçon d'Histoire donnée par Prokesch à l'Aiglon accentue l'influence néfaste des deux passés historiques dont le drame se nourrit. Metternich "parfait» l'éducation du duc en lui apprenant une Histoire tronquée, elliptique, qui évite toute allusion à Napoléon $\mathrm{I}^{\mathrm{er}}$. Cette leçon a l'effet inverse de ce qui était escompté. Au lieu de cacher au jeune homme l'histoire de son père, elle ne fait que la raviver car l'Aiglon, curieux de connaitre les exploits paternels, dort " sous un dôme d'Histoire ", rêvant, d'après les lectures qu'il a faites en autodidacte, au glorieux passé historique de l'Empire ${ }^{30}$. Dès ce moment, le rêve entrave l'action. «Oh ! vouloir à l'histoire ajouter des chapitres, / Et puis n'être qu'un front qui se colle à des vitres! ", explique le jeune homme à Prokesch ${ }^{31}$.

23 Les deux sens du mot "histoire " sont interrogés dans la pièce : la science du passé, telle qu'en rendent compte les historiens, et le récit littéraire, imaginaire, fait par un romancier ou un poète qui serait libre d'agir à sa guise. Le manque d'ardeur qui détourne le duc de l'« histoire à faire » l'empêche également d'écrire, l'éloigne de cet accomplissement alternatif qu'est l'écriture. D'ailleurs, contrairement à Cyrano de Bergerac, qui met en scène sa vie en donnant lui-même les indications scéniques aux spectateurs et aux lecteurs, l'Aiglon laisse le soin à d'autres personnages et à des poètes plus compétents de préparer ses entrées. La jeune Thérèse de Lorget lit à haute voix un 
passage extrait de Lamartine lorsque le duc monte en scène, tout comme Sorismonde avait précisé l'entrée en scène de Bertrand d'Allamanon dans le deuxième acte de $L a$ Princesse lointaine. Les paroles de l'Aiglon n'étant que des hésitations, les didascalies de la pièce se résument, outre les nécessaires indications sur le décor, à ses mouvements d'âme et à ses caprices.

Dans plusieurs pièces, les indications scéniques sur le décor montrent l'ouverture de la scène à d'autres horizons et attestent de la nécessité d'expansion de la poésie mais aussi, une nouvelle fois, de l'interpénétration de la poésie et des indications scéniques. Comme la poésie est dite, affirmée, dans Cyrano de Bergerac, La Princesse lointaine et Chantecler, et non pas réprimée comme dans L'Aiglon, elle s'élance vers le spectateur, outre les moyens déjà cités, par le biais du décor. Ce décor est échafaudé de manière à créer ce que Rostand nomme une "perspective ». Sur la mise en scène de Chantecler, il explique :

Enfin l'idée de mon décor est ceci : un effet de perspective comme pour une voie Appia: donner la sensation qu'une petite allée de jardin potager est pour les volailles une voie immense ; traiter la perspective comme si on voulait faire le décor des Champs-Élysées ; l'allée finit par être au premier plan large comme la scène, et donne ainsi beaucoup de place. On peut faire arriver autant d'allées qu'on veut sur cette allée, et avoir ainsi des dégagements des deux côtés. ${ }^{32}$

L'allusion explicite à la voie appienne permet l'ouverture du spectacle vers l'extérieur, le rayonnement de la mise en action qu'est le poème de Chantecler. L'allée, qui aboutit au premier plan, recueille le contenu de l'action et emporte la poésie vers des horizons différents. Dès cet instant, la fable de Chantecler acquiert la même ouverture spatiale que celle de La Samaritaine ou de La Princesse lointaine.

Même dans Cyrano de Bergerac, la conception des actes prend la même forme que celle de Chantecler. Chacun des décors est conçu pour rayonner vers l'extérieur. Rostand ne cesse d'élaborer une "perspective de fond", terme que l'on retrouve dans la majeure partie des didascalies en début d'actes, mais ne perd toutefois pas de vue le «ciel» également évoqué. Il n'y a pas que le premier acte de Cyrano de Bergerac, se déroulant à l'Hôtel de Bourgogne avec ses nombreuses galeries, qui crée un effet de perspective dans lequel peut s'immiscer le monde extérieur du public sur la scène et du public dans la salle. Elle permettra, par le jeu des mises en abîme, à l'univers entier d'assister à la naissance de la poésie, issue d'une voix venant d'ailleurs et qui se matérialise en geste didascalique. Dans la rôtisserie de Ragueneau, au deuxième acte de la pièce, se trouve au second plan: «[u]ne galerie de bois, faisant suite à l'escalier, [qui] semble mener à d'autres petites salles analogues » où la vie suit son cours, première échappatoire de la poésie vers le monde. Même dans la scène du balcon, classique exemple de huis clos, de conversation intime entre les amants, il y a $"$ [p]erspectives de ruelles. ${ }^{33}$ Les indications scéniques du quatrième acte, quant à elles, précisent: "Au fond, talus traversant toute la scène. Au-delà s'aperçoit un horizon de plaine : le pays couvert de travaux de siège. Les murs d'Arras et la silhouette de ses toits sur le ciel. $»^{34}$ Les didascalies du cinquième acte, encore, expliquent: "Tout le fond du théâtre est traversé par une allée de marronniers qui aboutit à droite, quatrième plan, à la porte d'une chapelle entrevue parmi les branches. À travers le double rideau d'arbres de cette allée, on aperçoit des fuites de pelouses, d'autres allées, des bosquets, les profondeurs du parc, le ciel. $»^{35}$ Cette description du décor insiste sur l'expansion, sur la perspective que prendra la création du poème auquel participent les gestes au-delà du «double rideau d'arbres » que représente le théâtre. La description et la disposition du décor 
permettent ainsi au personnage poétique de lancer sa poésie vers l'extérieur, au mépris de l'étroitesse mécanique et physique de la scène. Les indications scéniques fonctionnelles concernant le décor font exploser les limites du théatre en copiant les méthodes romanesques infinies de la description. Le théâtre pour Rostand se rapproche d'un roman qui mettrait en valeur la poésie comme l'expression d'une "attitude » réactive face à la vie, "attitude» située dans une certaine atmosphère que doit comprendre le spectateur.

Contrairement aux symbolistes, qui, à dessein, l'ont toujours entourée de mystère, la création poétique apparaît clairement et presque de manière naturaliste chez Rostand. Ce faisant, le spectateur peut l'appréhender dans toute sa simplicité. Il peut comprendre non plus la "condition " d'un poète, telle qu'illustrée dans le Chatterton d'Alfred de Vigny, mais bien l'« attitude » combative avec laquelle un homme de lettres envisage son métier.

\section{Conclusion}

Ainsi, nous pouvons conclure que Rostand a recours à la didascalie intérieure et extérieure aux discours des personnages pour mettre en scène la manière dont se crée un poème et la manière dont travaille un poète. C'est ainsi que, par la concomitance des gestes didascaliques et des paroles poétiques, il fait apparaître une " attitude " propre à la figure du poète. Le personnage au comportement bien défini évolue au sein d'un décor particulier, précisé par les indications scéniques en début d'actes. Le fond de scène, par l'apport d'une perspective, augmente la portée de cette "attitude " toute poétique auprès du public. Les travaux de Rostand laissent présager l'intérêt porté aux didascalies par les dramaturges modernes.

\section{NOTES}

1. Nous entendons par didascalies «tout texte [...] non prononcé par les acteurs et destiné à éclairer pour le lecteur la compréhension ou le mode de présentation de la pièce ». Elles comprennent le nom des personnages (didascalies initiales), les indications de leurs entrées et de leurs sorties, la description des lieux, la notation pour le jeu (didascalies fonctionnelles) et le discours rapporté (didascalies internes). Patrice Pavis, «Indications scéniques » dans Dictionnaire du théâtre, Paris, Dunod, 1996, p. 172-173.

2. Edmond Rostand, Chantecler, présentation par Philippe Bulinge, Paris, GF Flammarion, 2006, acte II, scène 1, p. 145.

3. Ibid., acte I, scène 1, p. 51-52.

4. Patrice Pavis, «Indications scéniques » dans Dictionnaire du théâtre, op. cit., p. 173.

5. Edmond Rostand, Cyrano de Bergerac, édition établie par Denis Roger-Vasselin, Classiques Hachette, 1997, acte II, scène 7, p. 135.

6. Pierre Mortier, «Je sais tout interviewe Edmond Rostand » dans Je sais tout, p. 438.

7. Lettre d'Edmond Rostand à Constant Coquelin (aîné) écrite en automne 1897. Edmond Rostand. L.A.S. [automne 1897], à Constant Coquelin ; 2 pages in-8 à son chiffre. Mn. 720 Inv. 99-80. 
8. Johann Wolfgang von Goethe, Faust, traduction d'Edmond Rostand, édition établie par Philippe Bulinge, Montreuil-sous-Bois, Éditions théâtrales, 2007, p. 13 et 15.

9. «Des broches tournent. Des pièces montées pyramident, des jambons pendent », précisent les didascalies du deuxième acte de Cyrano de Bergerac. Edmond Rostand, Cyrano de Bergerac, op. cit., acte II, p. 98.

10. Rosemonde Gérard, Edmond Rostand, Paris, Fasquelle Éditeurs, 1934, p. 13-14.

11. Pierre Mortier, op. cit., p. 438.

12. Edmond Rostand, Chantecler, op. cit., acte II, scène 5, p. 201.

13. Edmond Rostand, Discours de Réception à l'Académie française, Paris, Firmin-Didot et C ${ }^{\mathrm{ie}}, 1903, \mathrm{p}$. 3.

14. Edmond Rostand, Cyrano de Bergerac, op. cit., acte I, scène 2, p. 66-67.

15. Ibid., acte II, scène 10, p. 155.

16. Edmond Rostand, Chantecler, op. cit., acte II, scène 3, p. 168-169.

17. Edmond Rostand, Cyrano de Bergerac, op. cit., acte I, scène 4, p. 60.

18. Ibid., acte I, scène 4, p. 55-59.

19. Edmond Rostand, «A Sarah » dans Le Cantique de l'Aile, Paris, Charpentier et Fasquelle, 1922, p. 85.

20. Ibid., " A Coquelin », p. 90.

21. Lettre d'Edmond Rostand à Constant Coquelin (aîné) écrite en automne 1897. Edmond Rostand. L.A.S. [automne 1897], à Constant Coquelin ; 2 pages in-8 à son chiffre. Mn. 720 Inv. 99-80.

22. Edmond Rostand, Chantecler, op. cit., acte I, scène 2, p.

23. Edmond Rostand, Chantecler, op. cit., acte II, scène 3, p. 172.

24. Edmond Rostand, La Samaritaine, op. cit., deuxième tableau « La Porte de Sichem », scène 3, p. 84

25. Edmond Rostand, Chantecler, op.cit., acte I, scène 2, p. 69, 72, 74, acte II, scène 3, p. 167, 174, 177 et acte III, scène 4, p. 258, acte II, scène 3, p. 178, acte III, scène v, p. 263.

26. Edmond Rostand, Cyrano de Bergerac, op. cit., acte II, scène 8, p. 140.

27. Edmond Rostand, Chantecler, op. cit., acte I, p. 51.

28. Edmond Rostand, L'Aiglon, acte I « Les Ailes qui poussent », op. cit., p. 31.

29. Ibid., acte II « Les Ailes qui battent », p. 119.

30. Edmond Rostand, L'Aiglon, acte II « Les Ailes qui battent », scène 4, op. cit., p. 138.

31. Ibid., acte II « Les Ailes qui battent », scène 4, p. 139.

32. Article de L'Illustration théâtrale publié par Jacques Lorcey dans son Edmond Rostand, t. III, op. cit., p. 391.

33. Edmond Rostand, Cyrano de Bergerac, acte II « La Rôtisserie des poètes », op. cit., p. 97-98.

34. Ibid., acte IV « Les Cadets de Gascogne », p. 231.

35. Ibid., acte V « La Gazette de Cyrano », p. 299. 